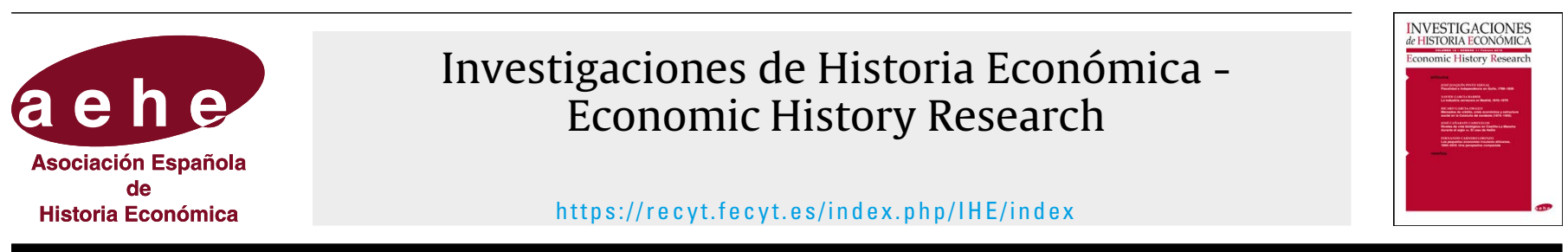

Artículo

\title{
Evolución de los salarios reales por cualificación en Uruguay, 1918-2009
}

\author{
María Camou* \\ Universidad de la República (Uruguay) (ID \\ Silvana Maubrigades \\ Universidad de la República (Uruguay) ID
}

\section{INFORMACIÓN DEL ARTÍCULO}

\section{Historia del artículo:}

Recibido: 18 de septiembre de 2020

Aceptado: 5 de octubre de 2021

On-line: 12 de noviembre de 2021

\section{Códigos JEL:}

$\mathrm{J} 40$

$\mathrm{J} 24$

$\mathrm{J} 31$

N36

Palabras clave:

\section{Salarios}

Premio por cualificación

Desigualdad salarial

Uruguay

\section{R E S U M E N}

Esta investigación proporciona nueva evidencia sobre la evolución de los salarios reales por cualificación en Uruguay entre 1918 y 2009 y su vínculo con los cambios en la demanda y oferta derivados de las etapas de desarrollo económico, así como del impacto de los cambios institucionales ocurridos en el período. Este trabajo mejora los datos existentes sobre la evolución de los salarios en Uruguay, proporcionando nuevas series de trabajadores cualificados, semicualificados y no cualificados. En el análisis se revela que los cambios en la demanda y los aspectos institucionales son determinantes en la evolución de los salarios por cualificación. La brecha salarial por cualificación no fue constante y, si bien se redujo hacia mediados del siglo xx, esta tendencia se revierte a partir de los años sesenta.

\section{Evolution of wages by skill level in Uruguay, 1918-2009}

\section{A B S T R A C T}

This research provides new evidence on the evolution of wages by skill level in Uruguay between 1918 and 2009. Furthermore, it explores the link with changes in demand and supply caused by economic development stages, as well as the impact of institutional changes that occurred during the period. This work improves the existing data on the evolution of wages in Uruguay, providing new series for skilled, semi-skilled and unskilled workers. The analysis reveals that changes in demand and institutional aspects are determining factors in the evolution of wages by skill level. The skill-based wage gap was not constant and, although it narrowed towards the middle of the 20th century, it widened beginning in the 1960s.

@ 2021 Asociación Española de Historia Económica

Skill premium

Wage inequality

Uruguay

\footnotetext{
* Autora para correspondencia. Correo electrónico: maria.camou@cienciassociales.edu.uy (María Camou). 


\section{Introducción}

El objetivo principal de este trabajo es presentar los resultados obtenidos en la construcción de una canasta de salarios por cualificación que dé cuenta de su evolución durante los diferentes modelos económicos en Uruguay a lo largo del siglo xx. Al mismo tiempo, integramos una interpretación de qué factores inciden en las diferencias observadas entre los niveles de cualificación salarial.

La investigación sobre la evolución en el largo plazo de los salarios en Uruguay ha sido limitada hasta el momento, debido a la falta de fuentes para su reconstrucción. Existen series de salarios de distintas ramas y ocupaciones para distintos períodos, pero como antecedente de largo plazo solo se cuenta con una serie de salarios para trabajadores de la construcción no cualificados para los años 1879-1996 (Bértola et al., 1999) ${ }^{1}$. Sobre la base de esta serie se ha construido una interpretación de la evolución de los salarios reales que abarca tres períodos principales: $1870-1930$, con un lento crecimiento de los salarios reales y el PIB per cápita; el período de industrialización (1930-1970), con un crecimiento significativo del PIB per cápita y un aumento aún mayor de los salarios reales, y un período final, donde se interrumpe esta tendencia y se produce una fuerte reducción de los salarios reales en un contexto de crecimiento del PIB per cápita. A partir de 1985, se inicia una lenta recuperación salarial.

Como parte central de nuestra investigación, realizamos un aporte a los datos existentes al construir series de diferenciales salariales entre trabajadores cualificados, semicualificados y no cualificados, urbanos y rurales, para el período 1918-2009. También elaboramos una serie específica de largo plazo para trabajadores rurales no cualificados. La selección de este período responde a la disponibilidad de datos requeridos para la construcción de estas series y abarca las distintas etapas del desarrollo tecnológico y económico del Uruguay.

El documento comienza con una breve presentación de nuestro enfoque teórico para el análisis del tema y su relevancia. En segundo lugar, presentamos la estrategia llevada adelante para construir nuestra base de salarios reales en el largo plazo y en períodos específicos de la economía uruguaya. Este punto es particularmente importante porque explicitamos las definiciones metodológicas utilizadas para la construcción de diferentes canastas de ocupaciones por cualificación para varios sectores. Esto implica que el salario promedio estimado para cada nivel de cualificación incluye ocupaciones cambiantes a lo largo del período. Esta decisión se basa en la idea de que el conjunto de ocupaciones escogidas debe reflejar los cambios en las estructuras del mercado laboral en el largo plazo. Un siguiente apartado presenta la evolución de los salarios no cualificados y su relación con el crecimiento económico en el largo plazo; así, se pretende contextualizar las principales etapas de crecimiento económico en Uruguay que abarca el período analizado. Partiendo de esta periodización, presentaremos nuestros resultados: la nueva serie de trabajadores cualificados, semicualificados y no cualificados para 1918-2010 en Uruguay. Para analizar la evolución de los distintos niveles salariales consideraremos los efectos de la oferta y la demanda,

\footnotetext{
${ }^{1}$ Esta serie fue elaborada en el marco de un proyecto de comparaciones internacionales que utilizó esta categoría de trabajadores para su comparabilidad.
}

así como los cambios institucionales en los distintos subperíodos. Finalmente, resumimos los resultados preliminares y dejamos algunas preguntas abiertas para futuras investigaciones.

\section{Marco teórico}

La abundante literatura en torno a los principales determinantes en la evolución de las diferencias salariales por cualificación, ha mostrado una gran diversidad en las distintas etapas y estrategias de crecimiento. La investigación sobre los casos particulares ha demostrado la inexistencia de un patrón único a lo largo del tiempo y en las distintas sociedades. En este sentido, en esta investigación optamos por un enfoque de largo plazo que permita identificar patrones cambiantes. A modo de síntesis, presentamos tres líneas de interpretación que han predominado en la literatura referente al tema. Aunque muchos de los autores citados utilizan explicaciones y modelos multivariados, sus explicaciones principales se organizan dentro de marcos que explicamos a continuación.

La primera interpretación enfatiza los cambios en la demanda de trabajo: principalmente destaca el rol del cambio tecnológico en el aumento de la demanda relativa de mano de obra cualificada (Acemoglu, 2003). Según Galor y Tsiddon (1997), en una etapa inicial el ciclo de innovación tecnológica tiende a aumentar la desigualdad salarial, ya que premia las cualificaciones (entendidas como experiencia o conocimiento no curricular), pero una vez que estas innovaciones se difunden y se vuelven más accesibles, la desigualdad tiende a disminuir. Las diferentes etapas del proceso de industrialización marcan distintos niveles de demanda de trabajo cualificado, incidiendo en las remuneraciones de este tipo de trabajo. La complementariedad entre cambio tecnológico, intensidad del capital y demanda de trabajo cualificado aparece en Estados Unidos, según algunos autores, a comienzos del siglo xx con la incorporación de la electricidad. En la etapa anterior habría existido un proceso de descualificación de la mano de obra en la industria, pero no en la economía en su conjunto (Katz y Margo, 2013). Profundizando en este supuesto, la demanda de trabajo cualificado dependería directamente de la intensidad del cambio tecnológico y la inversión en capital de las distintas ramas de la industria y su peso en la economía en su conjunto. Siguiendo con la experiencia de Estados Unidos, en una etapa posterior y con un mayor desarrollo y diversificación productiva, la evidencia indica un incremento en las desigualdades salariales desde la década de 1970. Estos resultados son atribuidos principalmente a nuevos requerimientos en la demanda de trabajadores, asociados estos con el cambio tecnológico en el marco de un nuevo paradigma basado en el conocimiento y la información y una escasez relativa en la oferta de trabajo específica requerida (Mincer, 1996). Ampliando el potencial explicativo de los cambios en la demanda, Anderson (2001) señala que, aunque no hay una explicación única para lo ocurrido entre la primera globalización y hasta la primera guerra mundial, la demanda de trabajo cualificado creció como resultado del cambio tecnológico en Europa y América y condujo a un aumento en los salarios de trabajadores cualificados. Mientras tanto, la disminución de la dispersión salarial a partir de la Primera Guerra Mundial en todos los países fue el resultado de un aumento en la demanda de trabajadores no cualificados y cambios intraindustriales en el empleo.

La segunda interpretación enfatiza los cambios en la oferta de trabajo, principalmente enfocada en el rol de la expansión 
de la educación en la caída del diferencial salarial. Desde una perspectiva de largo plazo que comprende varios siglos, Van Zanden (2009) encuentra evidencia de la correlación entre la caída del premio por cualificación y el crecimiento económico. Para este autor, el bajo nivel del premio por cualificación en la Europa del Norte sería un indicador del buen funcionamiento de las instituciones de formación de capital humano que habría otorgado una ventaja comparativa clave a esta región.

Para un período más reciente, los procesos de modernización y expansión de la educación y los cambios en los sistemas de capacitación de los trabajadores habrían llevado a incrementar la oferta de trabajadores cualificados. Goldin y Katz (2008) relacionan la disminución de la brecha salarial en los Estados Unidos durante la primera década del siglo xx con factores predominantemente de oferta: el aumento relativo en el número de trabajadores educados y una demanda más estable de trabajadores cualificados. Los autores identifican dos fases en la desigualdad: de 1915 a 1950, la prima salarial de los universitarios cae drásticamente, mientras que entre 1950 y 1980 aumenta rápidamente. En el primer período la educación habría ganado la carrera contra la tecnología, mientras que en el segundo la ralentización de la expansión educativa, no acompasada a las demandas del crecimiento intensivo en conocimiento, habría provocado el aumento del premio por cualificación. Asimismo, estos autores consideran otros factores como la incorporación masiva de las mujeres al mercado de trabajo y la inmigración para explicar el volumen y las características de la oferta de trabajo (íd.). En una mirada más amplia, Lewis (1954) argumenta que en las primeras etapas del proceso de industrialización se produce un aumento de oferta de mano de obra no calificada como consecuencia de la migración interna del medio rural al urbano. Este efecto, identificado en diferentes momentos históricos en los países desarrollados y los países en vías de desarrollo, genera una reserva de trabajadores que impacta en la presión a la baja de salarios de este tipo de trabajadores, contribuyendo así a un aumento de la desigualdad.

Autores como Williamson (1996), Wood (1998) y Anderson (2001) sostienen que la inmigración y la globalización tuvieron impactos diferenciados sobre la brecha salarial entre trabajadores cualificados y no cualificados del Viejo y Nuevo Mundo. En particular, Williamson argumenta que durante la primera globalización, entre 1870 y 1914, el aumento del comercio y la migración masiva de trabajadores de Europa hacia América, en su mayoría no cualificados, redujo la oferta en sus lugares de origen al tiempo que la aumentaron en el Nuevo Mundo, donde eran escasos. Esto causó una disminución de la desigualdad en Europa y su aumento en América y otros países receptores de migrantes. Luego, durante el período 1914-1960, la disminución del comercio y la migración revirtió este proceso. En esta misma línea, los resultados de la investigación de Federico et al. (2021) para Italia en el período 1861-1913 señalan la importancia de la emigración en la reducción de la oferta de trabajadores no cualificados -seguido en segundo lugar por el avance de la educación y la conflictividad laboral- en la caída del premio a la cualificación contrarrestando otros factores como el cambio estructural y la incorporación de tecnología.

La tercera línea explicativa que aquí consideraremos, si bien no niega la importancia de los factores de oferta y demanda, pone el acento en los factores institucionales (Rodgers, 1994). El sistema de remuneraciones es entendido en el marco de las teorías institucionalistas y regulacionistas como un sistema complejo de relaciones, que engloba no solamente el pago y las horas de trabajo, sino el compromiso del trabajador, la intensidad y el ritmo de trabajo, su relación a lo largo del tiempo con la empresa, su posición social, la forma en que se evalúan sus capacidades, etc. Desde un enfoque regulacionista (Boyer, 1994), la relación entre trabajo y acumulación, en sus diferentes componentes, aparece mediada por una serie de instituciones formales, como los sindicatos, empresas o legislación laboral y otras instituciones que se encuentran contenidas de forma implícita en las formas de control del trabajo, en las formas de distribución de los salarios entre personas de diferente sexo, edad u ocupación, en los mecanismos de salida y entrada del mercado de trabajo, etc. Estas instituciones del mercado de trabajo, socialmente construidas, admiten una gran diversidad de vínculos entre salario y empleo y, por lo tanto, no responden a una hipótesis de sustitución mecánica entre trabajo y capital. Todos estos aspectos antes mencionados son objeto de transacción y conflicto, expresándose a través de las remuneraciones. En el largo plazo, las instituciones laborales, a través de mecanismos como el salario mínimo, la participación de los sindicatos en la fijación del salario y la protección a los sectores discriminados, tienden a fortalecer la posición de los trabajadores no cualificados y, por lo tanto, a disminuir el peso relativo de la retribución según la cualificación.

Otros autores como Betrán y Pons (2003) sostienen, basándose en un análisis de la evolución del premio por calificaciones en cinco países en el período 1870-1930, que la globalización y los cambios en la demanda derivados del avance tecnológico solo pueden explicar parte de las diferencias entre estos países. Factores institucionales como el grado de conflictividad de los trabajadores y las políticas migratorias contribuyen a entender estos procesos, siendo además un factor clave en la adaptación entre demanda y oferta de trabajo a través de las mejoras de las condiciones de trabajo y la capacitación de los trabajadores.

Para un período más reciente 1980-2001, Card, Lemieux y Ridde (2004) presentan evidencia contundente de la incidencia de la sindicalización en las desigualdades salariales en Estados Unidos, Reino Unido y Canadá. Para los tres países se comparan niveles de dispersión salarial (controlando por otros factores como nivel de cualificación, sector, etc.) entre sectores sindicalizados y no sindicalizados, y se encuentra una menor dispersión entre los primeros, aunque solo para los hombres.

La riqueza del análisis de estas tres líneas explicativas y la posibilidad de que ellas se den en forma simultánea da cuenta de que el tema de las retribuciones salariales exige una explicación multicausal. Los estudios de caso, que aporten un análisis en profundidad sobre la combinación de estos factores, pueden ofrecer nuevos elementos en esta discusión, ya que el cambio tecnológico impacta en las diferencias salariales, pero también puede estar determinado por las características particulares del mercado de trabajo local (Grantham, 1994). Desde esta perspectiva, en esta investigación nos proponemos, en primer lugar, contribuir a esta discusión generando nueva evidencia sobre la evolución de los salarios por tipo de cualificación. Asimismo, presentaremos nuestras series en el marco de una interpretación de los resultados en el contexto de tres etapas del desarrollo económico en Uruguay. Nuestro análisis de largo plazo toma en cuenta, para cada uno de estos períodos, los factores de oferta y demanda y los cambios y quiebres institucionales que se producen. 


\section{Estrategia de análisis de la evolución salarial por cualificación en Uruguay en el largo plazo}

El antecedente más importante para Uruguay de una reconstrucción de largo plazo sobre esta temática es la serie de salarios de trabajadores de la construcción no cualificados,para el período 1870-2010 (Bértola et al., 1999). Posteriormente, las series de salarios promedio por sectores industriales para el período 1930-1969 fueron presentadas por Lara (2010), trabajo que tomó en consideración la reconstrucción de salarios para obreros y empleados de la industria para el período comprendido entre 1936-1947, construído por Millot et al. (1973). Otra investigación que abarca el período comprendido entre 1946 y 1955 se centró en la evolución de los salarios por ocupación acordada por los consejos salariales de esos años (Notaro, Fernández Caetano y Sörensen, 2012). En un proyecto anterior, Camou (2010) analizó la evolución de los salarios por cualificación para dos empresas industriales (textiles y empacadoras de carne) durante la ISI (Industrialización por sustitución de importaciones), y una de sus principales conclusiones fue que una disminución de la desigualdad podría detectarse durante este período. Para períodos más recientes se han realizado estimaciones parciales de evolución salarial de acuerdo con la cualificación de los trabajadores (Amarante y Arim, 2005; Bucheli, 1992), analizándose también el desfase entre la cualificación de los trabajadores y su inserción laboral (Espino, 2011).

En esta investigación nos propusimos abordar, de acuerdo con una reconstrucción basada en nuevas fuentes disponibles, el período comprendido entre 1918 y 2009. Para ello, reconstruimos en primer término una serie de salarios de largo plazo de trabajadores no cualificados que comprende una gama más amplia de ocupaciones. Esto representa un avance respecto de la preexistente, ya que aquella, por escasez de datos con los que se contaba, había recurrido para algunos años a la proyección por índice medio de salarios de la industria (Bértola et al., 1999).

Para profundizar este análisis a largo plazo de los diferenciales salariales de acuerdo a la cualificación de los trabajadores, elaboramos una canasta cambiante de categorías ocupacionales que cubriera todo el período estudiado. Para dar cuenta de las diferencias salariales por cualificación, y no contando hasta el momento con información confiable que vincule los niveles educativos de la población y el tipo de ocupación de los trabajadores, construimos series para el período 1918-2009 que incluyen salarios no cualificados, semicualificados y cualificados, partiendo de los salarios de diferentes grupos de ocupación. Procuramos que estas series abarcaran los grupos de ocupación definidos por la OIT: trabajadores cualificados (empleadores, gerentes y profesionales); trabajadores no cualificados de cuello azul y de cuello blanco, y no cualificados (trabajadores rurales y urbanos no cualificados, incluidos los empleados domésticos $\mathrm{y}$ vendedores ambulantes). La lista de ocupaciones elaborada fue modificándose a lo largo del tiempo para representar con fidelidad la realidad del mercado de trabajo uruguayo en las diferentes etapas de desarrollo económico. Con ello procuramos considerar el impacto de los cambios tecnológicos y la demanda del mercado a lo largo del período analizado. A modo de ejemplo, en el período de industrialización del Uruguay los tejedores ocupaban un lugar relevante como trabajadores semicualificados dentro de la estructura ocupacional; sin embargo, hacia fines del siglo xx la rama textil estaba ya desapareciendo de la estructura productiva del país y el proceso de producción se transformó hacia la elaboración de productos de menor valor agregado, por lo que la demanda de tejedores disminuyó, al igual que sus salarios relativos.

El trabajo de reconstrucción de las series salariales por categoría ocupacional requirió el uso de diversas fuentes y la respectiva homogeneización de las mismas. Para el período 19181981 se utilizaron como fuentes diversos archivos comerciales, presupuestos estatales y la Encuesta de Octubre de la OIT (parte I y II). También se utilizaron para completar vacíos series de salarios mínimos de investigaciones previas que fueron aplicadas para proyectar la evolución de los salarios. Para el período (1981-2010) se utilizó como base los datos de la Encuesta de Hogares generada por el Instituto Nacional de Estadística.

Para el período 1918-1957 se procesó información de los archivos de dos industrias importantes de Uruguay: la empresa textil Campomar y la planta empacadora de carne Swift. Estas fuentes permitieron acceder a información sobre salarios por ocupación, que no existía a nivel macro. El archivo Campomar comprende una gran parte de la documentación de esta empresa desde su fundación en 1898 hasta su cierre final en los años noventa del siglo xx. En el caso de Campomar, los registros salariales individuales de toda la fuerza laboral de la planta se cuentan durante un trimestre de cada año durante el período 19181951. Cada registro contiene nombre, número de registro, ocupación, número de horas trabajadas, total ganado, suplementos, licencias y descuentos. El archivo Swift utilizado en esta investigación cubre los registros de personal de los trabajadores para el período 1918-1957. El universo de datos recopilados entre ambas compañías incluye a todos los trabajadores, lo que significa un promedio de aproximadamente 6000 trabajadores por año para el período 1913-1957. Los trabajadores de Swift y Campomar representaban entre el $5 \%$ y $7 \%$ de la fuerza laboral industrial de Uruguay, dependiendo de las variaciones anuales. Este universo es extremadamente amplio y asegura un alto grado de fiabilidad a los resultados obtenidos.

Los salarios públicos cualificados se reconstruyeron sobre la base de los presupuestos de gastos generales de la República del Uruguay, que proporcionan información anual sobre los salarios de todas las dependencias estatales. Hasta principios de la década de 1950, los salarios experimentaron pocos cambios en los períodos interpresupuestales, pero hacia el final del período el proceso inflacionario resultó en un aumento de las leyes y decretos que modificaban mediante enmiendas la ley de presupuesto.

La evolución de los salarios rurales en el período 1918-1960 se pudo estudiar gracias a los libros de contabilidad de Bodega Santa Cruz. Para el período 1960-1981, la serie que abarca la evolución salarial de los trabajadores rurales se reconstruyó sobre la base de los libros de sueldos y salarios de la estancia San Pedro del Timote, ubicada en la cercanía de la estación de trenes de Cerro Colorado en el departamento uruguayo de Florida. Este gran establecimiento, propiedad de Alberto Gallinal, era conocido por su alta calidad de ganado y su volumen de producción

La elaboración de los datos de salario por categoría ocupacional utilizó el valor del jornal básico a precios nominales, sin considerar aportes sociales u otro tipo de complemento salarial. Los resultados se convirtieron posteriormente a valores mensuales y fueron expresados en precios constantes de 1970. Para deflactar los datos se utilizó el IPER CÁPITA obtenido de Bértola et al. (1999) entre 1918-1980 y del INE entre 1980-2009.

A continuación, se presenta una descriper cápitaión exhaustiva de las fuentes utilizadas para cubrir el período estudiado. 
Tabla 1.

Fuentes y métodos para la construcción de la serie

\begin{tabular}{|c|c|c|c|c|c|c|c|c|}
\hline \multirow{2}{*}{$\begin{array}{l}\begin{array}{l}\text { Sub-conjunto } \\
\text { de trabajadores }\end{array} \\
\text { Categorías } \\
\text { incluidas }\end{array}$} & \multirow{2}{*}{$\begin{array}{l}\text { No calif. rural } \\
\text { Peón rural }\end{array}$} & \multirow{2}{*}{$\begin{array}{l}\text { No calif. urbano } \\
\text { Peón no } \\
\text { cualificado }\end{array}$} & \multicolumn{3}{|c|}{ Semi calif. priv. } & \multirow{2}{*}{\begin{tabular}{|l}
\multicolumn{1}{|c|}{ Calif. pub. } \\
$\begin{array}{l}\text { Agrimensor, } \\
\text { docente de } \\
\text { secundaria }\end{array}$
\end{tabular}} & \multicolumn{2}{|c|}{ Calif. priv. } \\
\hline & & & $\begin{array}{l}\text { Tejedores, } \\
\text { reparadores de } \\
\text { máquinas, } \\
\text { maquinistas de } \\
\text { garaje, } \\
\text { maquinistas, } \\
\text { oficiales }\end{array}$ & $\begin{array}{l}\text { Trabajadores } \\
\text { industriales } \\
\text { (panaderos, } \\
\text { tejedores, } \\
\text { tipógrafo, } \\
\text { mecánico) }\end{array}$ & $\begin{array}{l}\text { Trabajadores } \\
\text { de servicios, } \\
\text { vendedores } \\
\text { de tiendas, } \\
\text { agricultores y } \\
\text { trabajadores de } \\
\text { la agricultura, } \\
\text { operadores y } \\
\text { artesanos de } \\
\text { operadores y } \\
\text { ensambladores } \\
\text { mecánicos, de } \\
\text { plantas y } \\
\text { máquinas }\end{array}$ & & $\begin{array}{l}\text { Administrativo } \\
\text { jefe; } \\
\text { trabajadores } \\
\text { de cuello } \\
\text { blanco } \\
\text { (asistente de } \\
\text { laboratorio, } \\
\text { vendedores, } \\
\text { cajeros banca) }\end{array}$ & $\begin{array}{l}\text { Trabajadores } \\
\text { cualificados. } \\
\text { Profesionales } \\
\text { y técnicos de } \\
\text { acuerdo al } \\
\text { código CIUO } \\
(1 \text { y } 2)\end{array}$ \\
\hline Fuentes & $\begin{array}{l}1(1917-1956) \\
3(1961-1975) \\
4(1975-1981) \\
10(1981-2009)\end{array}$ & $\begin{array}{l}5,6(1918-1957) \\
9(1927-1973) \\
10(1981-2009)\end{array}$ & 5, 6 (1918-1957) & $9(1964-1981)$ & 10 (1981-2009) & $8(1918-1955)$ & $4(1935-1949)$ & $9(1964-1981)$ \\
\hline $\begin{array}{l}\text { Método de } \\
\text { agregación }\end{array}$ & $\begin{array}{l}\text { Promedio } \\
\text { simple jornal }\end{array}$ & $\begin{array}{l}\text { Promedio } \\
\text { simple jornal }\end{array}$ & $\begin{array}{l}\text { Promedio } \\
\text { simple jornal }\end{array}$ & & & $\begin{array}{l}\text { Promedio } \\
\text { ponderado } \\
\text { (agrimensor } \\
30 \% \text {, profesor } \\
70 \% \text { ) }\end{array}$ & $\begin{array}{l}\text { Promedio } \\
\text { simple jornal }\end{array}$ & \\
\hline Interpolación & a) $1956-1961$ & b) $1968-1981$ & c) $1952-1964$ & & & & & \\
\hline
\end{tabular}

Fuentes:

Establecimiento Bodega Santa Cruz (FHE).

Establecimiento Estancia San Pedro del Timote (AGN).

Oficina de Estadísticas Agropecuarias (DIEA) del Ministerio de Ganadería, Agricultura y Pesca (MGAP).

Archivo Campomar y Soulas (FCS).

Archivo empresa Swift (FCS).

Ministerio de Obras Públicas. Presupuesto Nacional.

Encuesta Continua de Hogares (INE).

Presupuesto Nacional. Datos bianuales.

Oficina Internacional del Trabajo. Encuesta de Octubre de la OIT (parte I y II).

Interpolaciones según: no cualificado (Bértola et al., 1996); salario mínimo nacional (INE); oficial textil (Davrieux, 1970).

Nota: se utilizó la interpolación lineal para llenar el vacío entre dos puntos de datos.

\section{Evolución salarial y crecimiento económico en Uru- guay}

La evolución comparada del PIB per cápita y los salarios reales (no cualificados) muestra en el largo plazo diferentes escenarios (ver gráfico 1). Para el período comprendido entre 1918 y los inicios de la década de los años treinta, la dinámica de crecimiento del producto bruto fue mayor que la observada en los salarios. Tal resultado es consistente con una recuperación económica post Primera Guerra Mundial con dos ejes centrales y complementarios. Por un lado, una inserción internacional basada en la exportación de productos primarios orientada hacia los países europeos. Por otro, un proceso de incipiente industrialización, que cobró dinamismo durante la década de 1920, dedicado al procesamiento de las materias primas nacionales y a la sustitución de bienes perecederos (Jacob, 1981; Bértola, 2000; Bertino et al., 2001).

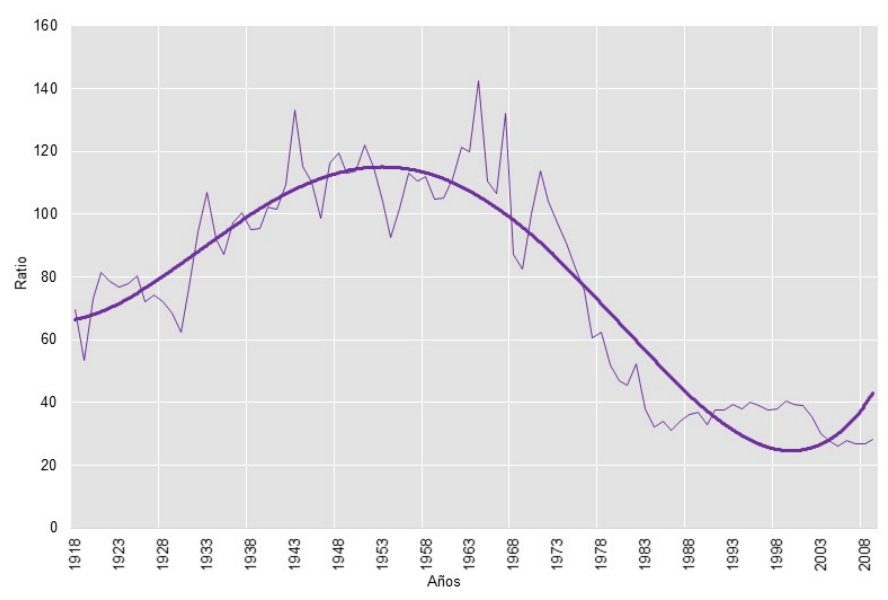

Gráfico 1. Ratio salario real no cualificado y PIB per cápita. Uruguay 19182009 (Índice $1970=100$ ).

Fuente: salarios reales (no cualificados): elaboración propia (ver tabla 1). IPER CÁPITA (Bértola et. al.,1999) e INE. PBI per cápita (Bértola, 2016). 
En igual período, parece que los trabajadores no cualificados han tenido escasa capacidad de incidencia en la fijación de los salarios, al tiempo que las condiciones laborales perdían relevancia en el discurso económico y político de la época (Jacob, 1984). De hecho, sería recién hacia finales de los años treinta, luego de que la economía local superara el impacto de la crisis mundial ocurrida en 1929, cuando la clase política expresaría su preocupación por las condiciones de vida de los trabajadores y se consolidaba un escenario de debate parlamentario en torno a la regulación del trabajo y la fijación de los salarios (Diario Oficial, 1939).

Desde principios de la década de 1930 se inició un proceso paulatino de industrialización impulsado por el Estado, que tendría su etapa de auge entre 1945 y 1957 (Bértola, 1991; Millot et al., 1973). Se implementaron en estos años políticas proteccionistas para los diversos sectores productivos del país, con un fuerte impulso a la sustitución de importaciones, sustentada en la importación de bienes de capital y en la construcción de un mercado interno fuertemente protegido y regulado. Los años comprendidos entre 1945 y 1968 serían un período en el que los ingresos salariales tuvieron una mayor participación en los resultados económicos en su conjunto (Siniscalchi y Willebald, 2018). La instauración de un régimen de regulación laboral, en donde trabajadores, empresarios y Estado entablaron un sistema de negociación colectiva de los salarios, generó las condiciones para que los logros económicos del período se distribuyeran en una forma menos desigual. La fijación acordada de salarios fue sostenible, en tanto los resultados económicos fueron positivos y el Estado asumió un rol articulador entre las distintas partes (Notaro, 2012).

Sin embargo, ya desde finales de la década de 1950, la economía comenzó un espiral descendente, en parte explicado por la caída drástica del valor de las exportaciones de bienes primarios (Bértola, 1991). Esta pérdida de divisas afectó rápidamente al andamiaje institucional montado por el Estado durante las dos décadas anteriores, en donde las divisas provenientes de las exportaciones eran redistribuidas al resto de la economía promoviendo con ello una industrialización fuertemente protegida. Esta política, que también estuvo orientada a garantizar niveles salariales que contribuyeron a sostener el mercado interno, empezó a ser desarticulada con el objetivo de revertir los efectos de la crisis económica.

En la puja distributiva que duró toda la década de 1960, en el marco de un estancamiento del PBI per cápita, los salarios no cualificados se vieron sujetos a fuertes oscilaciones. La conflictividad aumentó y con una inflación muy alta los resultados, que parcialmente podían obtener los trabajadores a través de los consejos de salarios, se volatilizaron rápidamente. Para 1968 , con un fuerte cambio de política económica, se iniciaría un proceso de caída ininterrumpida de los salarios reales, coincidente con la recuperación del producto (Melgar y Cancela, 1985; Davrieux, 1970).

El Gobierno de J. Pacheco Areco (1967-1971) implementó una variedad de medidas para restablecer el equilibrio de la balanza comercial y estabilizar los precios. Una de las medidas más controvertidas del momento fue la congelación de precios y salarios que se impuso en junio de 1968, que constituyó el primer paso hacia la reducción de los salarios reales, junto con la eliminación de la negociación colectiva de los mismos (Notaro, 1984). Será este un punto de inflexión a partir del cual se observa una evolución divergente entre salarios no cualificados y producto bruto interno, que se mantiene hasta finales de la década de 1980. Estas medidas se enmarcaron en lo que fue una respuesta al fracaso del modelo de industrialización dirigida por el Estado y el retorno a la estrategia de principios del siglo $\mathrm{xx}$, orientada al crecimiento impulsado por las exportaciones. Esta nueva etapa de apertura, que comenzó en la década de 1970, vino acompañada de un paquete de medidas económicas que fueron expandiéndose y abarcaron gran parte de la región latinoamericana. Las principales políticas económicas que se implementaron estuvieron centradas en la liberalización comercial y financiera, la privatización y la desregulación del mercado laboral, así como en el desmantelamiento de las políticas industriales proteccionistas del período anterior (CEPAL, 2018).

La puesta en práctica de este paquete de medidas para la economía local, con un fuerte componente concentrador y excluyente en el plano social (Olesker, 2001), fue posible mediante la instalación - al inicio del período - de una dictadura militar que se prolongó durante más de una década (19731984) La estrategia económica del Uruguay estuvo basada en el retorno a las exportaciones tradicionales, principalmente la exportación de ganado, pero la crisis del petróleo y las restricciones europeas a las importaciones se interpusieron. En ese contexto, el sector exportador se reorientó hacia exportaciones no tradicionales, basadas también en el procesamiento de bienes primarios con un escaso valor agregado, con mano de obra de baja cualificación y bajos salarios. Durante todo el período la caída salarial fue sostenida y el aumento del desempleo y un fuerte incremento del mercado laboral informal, especialmente a partir del inicio de la década de 1980, contribuyeron a mantener esta tendencia decreciente del valor trabajo (Buchelli, 1998; Notaro, 1984). Como resultado, los salarios reales promedio en 1984 representaron el 61,7\% del nivel alcanzado en 1970.

En los años posteriores a 1985, si bien se recuperó la democracia, se profundizaron las políticas económicas implementadas en la década anterior y esto condujo a la consolidación de un modelo liberal e inequitativo de puertas abiertas, característico de esta segunda globalización (Mordecki, 2017, 2015; Antía, 2001; Macadar, 1992). El modelo combinó exportaciones intensivas en recursos naturales a la región y otros mercados emergentes, con un modesto comercio intraindustrial principalmente con Argentina. En el transcurso de la última década del siglo xx, el país asistió a un desmantelamiento del sector industrial y un incremento del sector de los servicios fuertemente segmentado. El salario real (no cualificado), si bien mostró una recuperación moderada, no logró volver a los niveles anteriores a la crisis de los años sesenta (Espino, 2004).

Las características sobresalientes de esta continuidad económica de las últimas tres décadas analizadas fueron la creciente importancia relativa de las empresas en la determinación de las estrategias productivas y su correlato en la fijación de los salarios. Esto estuvo apoyado en una mayor flexibilidad laboral y en una pérdida de influencia del sector sindical (Amarante, 2005; Supervielle, 2000). Los niveles de desempleo se mantuvieron sin cambios e incluso empeoraron durante la década de 1990, a pesar de un aumento en el PIB per cápita (Espino, 2004; Marinakis, 2005). Las condiciones de trabajo precarias, la informalidad y el subempleo crecieron aún más, mientras que la protección social de los trabajadores por parte del Estado disminuyó significativamente en esa etapa (Marinakis, 2005; Filgueira, 2008). 


\section{Las principales determinantes en la fijación de los salarios por cualificación}

La estructura productiva de la economía ha condicionado fuertemente el crecimiento y composición del mercado laboral. Así, la base productiva del país y el motor de la inserción internacional del Uruguay, a lo largo de todo el período analizado, ha sido el sector agrario (Álvarez, 2015). Sin embargo, este sector se ha caracterizado por una escasa demanda de mano de obra en general y la fuerza de trabajo requerida ha sido de baja cualificación y con una marcada estacionalidad, especialmente en el sector agrícola. El excedente de mano de obra generado contribuyó a mantener los salarios del sector sistemáticamente bajos, especialmente en relación con los restantes sectores de actividad (ver gráfico 2). Esto ha convertido al sector primario en un fuerte expulsor de mano de obra, desde el medio rural a los centros poblados y especialmente a la capital del país, concentradora de la mitad de la población nacional desde los orígenes (Moraes, 2003).

En cambio, la evolución del mercado laboral para los restantes sectores de la economía ha tenido una trayectoria más cambiante a lo largo de los siglos xx y xxI, dependiente de los requerimientos productivos y tecnológicos y de las diferentes estrategias del crecimiento económico. Esto ha determinado que las diferencias salariales, según la cualificación de los trabajadores, puedan ser explicadas, en parte, en función de las demandas de trabajadores generadas por los diversos sectores de actividad y sus niveles de desarrollo. Por otro lado, los cambios ocurridos en la oferta de fuerza de trabajo, especialmente en materia educativa, también explican las trayectorias salariales y el premio a la cualificación. El impulso a la educación en el Uruguay destacó tempranamente al país en la región, trascendiendo esta inversión pública los requerimientos exclusivamente productivos (Bértola et al., 2010; Camou y Maubrigades, 2005). Finalmente, los cambios ocurridos en las políticas salariales en los distintos modelos de desarrollo antes descritos nos hablan de un peso desigual de las políticas estatales en los resultados en materia salarial: especialmente, los períodos con una fuerte influencia de la regulación salarial implican reducciones significativas de las brechas salariales por nivel de cualificación, como veremos a continuación.

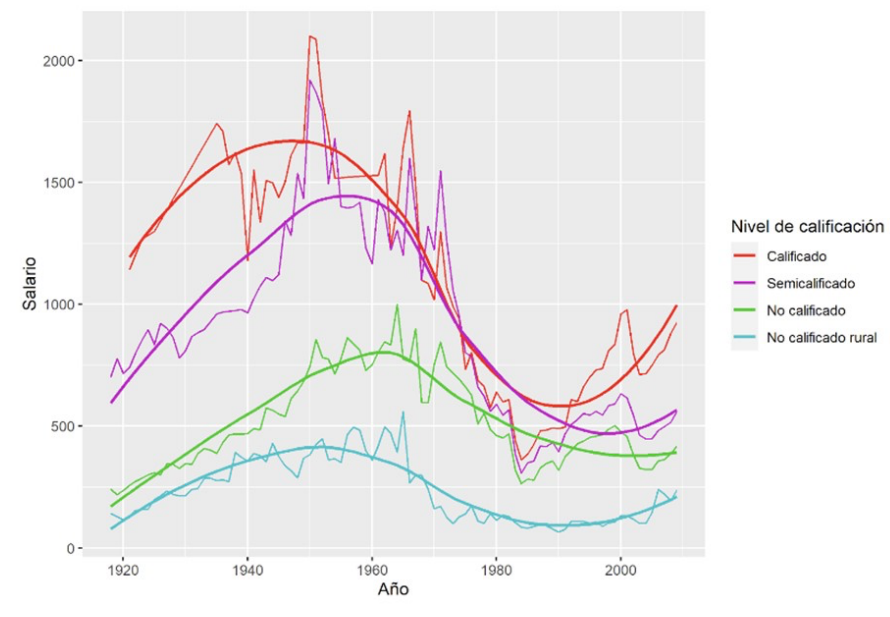

Gráfico 2. Evolución de los salarios reales por tipo de cualificación. Uruguay, 1918-2009 (pesos de 1970).

Fuentes: archivos de la empresa Campomar y Swift y presupuestos generales de gastos (1918-1980). Encuesta Continua de Hogares (1981-2009) (ver tabla 1). IPC: Bértola et. al. (1999) e INE.
Según la información disponible, entre 1918 y 1930 y en el marco de un proceso de industrialización temprana, la diferencia salarial según cualificación fue la más alta de todo el período analizado. Para estos años, el salario de los trabajadores cualificados fue aproximadamente cuatro veces mayor que el salario de los trabajadores no cualificados. Desde el lado de la demanda de trabajadores, esta estructura productiva poco diversificada generó una segmentación entre una fuerza de trabajo calificada, volcada a un crecimiento del sector de los servicios -especialmente en la Administración pública-y un sector de trabajadores poco cualificados que fueron insertándose en el sector secundario de la economía. Siguiendo los pasos de Galor y Tsiddon (1997), en esta etapa temprana de industrialización es esperable encontrar estas diferencias salariales en un contexto de escasa cualificación para el incipiente desarrollo de este sector productivo.

Por otro lado, los cambios en la oferta de mano de obra también contribuyeron a profundizar estas diferencias salariales. En el período 1918-1930 se contaba con una mano de obra local poco calificada dentro de un sector fabril en ascenso. El proceso migratorio del campo a la ciudad, ocurrido desde finales del siglo xIx, generó una fuerza de trabajo poco especializada y con una baja acumulación de años de estudio (Camou, 2020; Bértola y Bertoni, 2000). En igual período, el país recibió un fuerte flujo migratorio desde los países europeos (principalmente españoles e italianos), que contribuyeron a engrosar esta fuerza de trabajo poco cualificada y dispuesta a insertarse en un mercado laboral en ascenso (Mourat, 1969). Este incremento sostenido de la oferta de población en edad de trabajar contribuyó también a contener el aumento de los salarios, especialmente entre trabajadores semicualificados y no cualificados, que eran requeridos por el tipo de industrialización en curso, así como por un sector de los servicios con escasos puestos de trabajo de alta cualificación.

Desde principios de la década de 1930 , la reacción del país ante la Gran Depresión fue la implementación progresiva de una política destinada a industrializar la economía, reducir las importaciones de bienes manufacturados, aumentar el empleo y dinamizar el mercado interno. Este proceso de industrialización liderada por el Estado siguió una estrategia de acrecentar las barreras arancelarias para desalentar las importaciones de bienes finales y proteger a las nuevas empresas manufactureras, así como apoyar las importaciones de bienes de capital y tecnología de los países industrializados. El tipo de desarrollo industrial que se generó hizo un uso intensivo de mano de obra, pero con escasa cualificación y poca especialización. Durante este periodo crecieron en el Uruguay las industrias procesadoras de materias prima (alimentos, bebidas, textiles, ropa y cuero), pero también se logró un aumento significativo de otras industrias procesadoras de materias primas importadas orientadas al mercado interno (madera, metalurgia, productos químicos, refinación de petróleo y equipos eléctricos) (Bértola, 1991). Una mirada en conjunto del sector productivo mostraba cómo el peso relativo del sector agrario disminuye, al tiempo que la participación de los sectores manufacturero y de servicios, tanto en el empleo como en el PIB, se fortalecen (Ardente, Díaz y Rossi 2004; Bértola, 2005).

Si bien desde principios del siglo xx Uruguay tuvo una mejora en los niveles educativos de la población en edad de trabajar (ver gráfico 3), el tipo de desarrollo industrial promovió un proceso de aprendizaje en los lugares de trabajo más que requerimientos de enseñanza formal y técnica. El tipo de de- 
manda de mano de obra durante el proceso de industrialización, mucho más que los eventuales cambios en la oferta de trabajo cualificado, explica la evolución salarial y la reducción de las diferencias en los salarios relativos de trabajadores cualificados y no cualificados, así como entre trabajadores cualificados y semicualificados (Camou, 2010).

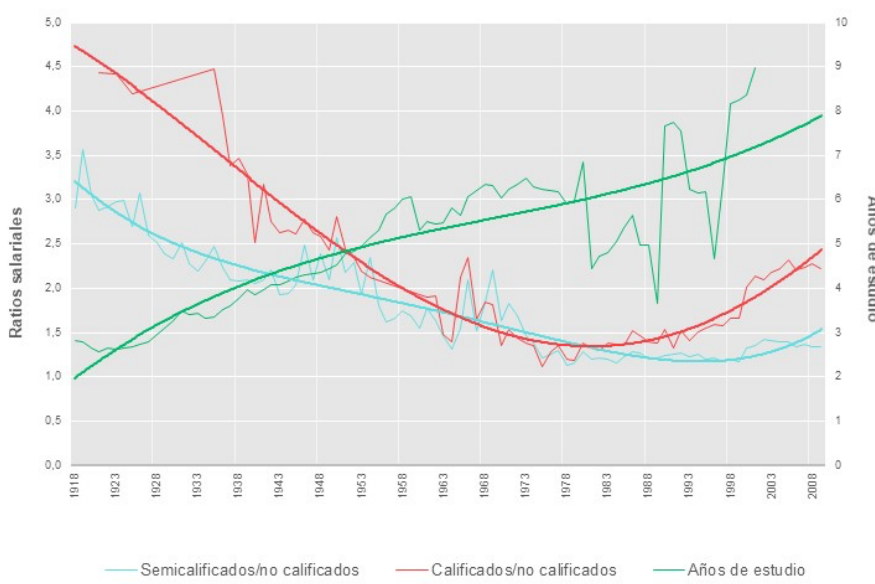

Gráfico 3. Evolución del nivel educativo y las ratios salariales por cualificación (Uruguay, 1918-2009).

Fuentes: para los salarios reales: archivos de la empresa Campomar y Swift y presupuestos generales de gastos (1918-1980). Encuesta Continua de Hogares (1981-2009). Educación: 1918-2000 (Bértola et al., 2010).

Pero durante este proceso de industrialización dirigida por el Estado, en los cambios ocurridos en los niveles salariales también tuvo un peso significativo la regulación salarial y el fuerte involucramiento de los trabajadores en la negociación colectiva (Notaro, 2012). La implementación, a partir de 1943, de convenios colectivos tripartitos (empresarios, trabajadores y Estado) para la fijación de salarios mínimos de los sectores industriales y de servicios en el ámbito privado, contribuyó al significativo descenso de las diferencias salariales entre los trabajadores de acuerdo al tipo de cualificación (ver gráfico 3). A la fijación negociada de salarios mínimos, se agregaron en este contexto un conjunto de políticas públicas identificadas con un Estado de bienestar adelantado para su época. No solo se amplió la negociación salarial y las políticas industriales, sino que además se expandió el gasto social y se introdujeron controles de precios para los productos alimenticios básicos y los alquileres. Cabe señalar que el mismo período de disminución de las diferencias salariales entre los trabajadores fue testigo de un proceso de aumento del gasto en educación y servicios sociales (Azar, 2012; Camou y Maubrigades, 2005). Esta combinación de factores contribuyó a una mejora significativa del salario real promedio general, coincidente con una caída general de la desigualdad (Bértola, 2005).

Una mención específica merece el comportamiento del sector agrario de la economía, donde se observa la poca incidencia de este clima de mejoras en los salarios rurales respecto al resto de los trabajadores. Este sector, si bien tuvo una moderada expansión económica, no modificó su baja demanda de mano de obra, además de haber mantenido un régimen de estacionalidad en el empleo de trabajadores, lo que desestimuló la permanencia de estos en el medio rural. Hay que señalar que los trabajadores rurales no estuvieron comprendidos den- tro del régimen de regulación salarial. El tipo de producción latifundista que caracteriza el agro uruguayo genera escasa demanda de mano de obra, lo que ha debilitado la capacidad de nuclear estos trabajadores y negociar sus retribuciones salariales (Piñeiro y Moraes, 2008).

Este conjunto de mejoras obtenidas en materia salarial comienza a mostrar sus debilidades en la década de 1960 . Una caída acelerada de los términos de intercambio a partir de 1957 derivó en una incapacidad económica para sostener la política proteccionista de fomento a la industrialización sustitutiva de importaciones (Bértola, 1991). Esto generó una desaceleración en el crecimiento del producto manufacturero, un déficit crónico en la balanza de pagos, un crecimiento acelerado de la inflación, elementos todos que promovieron también un aumento de la conflictividad social y política (Notaro, 1984).

La caída en la rentabilidad de los sectores productivos en general, pero especialmente en las ramas más dinámicas de la industria de sustitución de importaciones, generó una contracción en los niveles salariales (Bértola, 1991). Esto fue especialmente provocado por una pretendida reducción de los costos globales de la producción y por un ajuste significativo de los costos de la mano de obra. De acuerdo con los resultados de este trabajo, se observa en este período una caída ininterrumpida del salario en su conjunto, pero muy especialmente entre los sectores cualificados y semicualificados de la fuerza de trabajo. La reducción en la brecha salarial por cualificación, observada a partir de la década de 1960, se debió más a una caída de los niveles salariales de las ocupaciones más cualificadas que a mejoras en los niveles salariales inferiores, lo que daba cuenta de un empobrecimiento de las condiciones laborales, tanto desde el punto de vista cuantitativo como cualitativo.

Como se mencionó anteriormente, las reformas económicas iniciadas a partir de la década de 1970 no solo estuvieron marcadas por una política de apertura externa y de reprimarización de la economía, sino que implicaron el inicio de una flexibilización del mercado laboral y una desregulación de las condiciones de trabajo (Olesker, 2001; Notaro, 1984). El resultado fue una abrupta desaparición de todos los mecanismos de negociación salarial existentes hasta el momento. La reprimarización de la economía generó drásticos cambios en la demanda de mano de obra cualificada, así como una fuerte desprotección de los trabajadores y sus condiciones salariales (Olesker, 2001; Notaro, 1984). El crecimiento del desempleo también jugó su parte en la caída de los niveles salariales. Este trabajo encuentra que esto tuvo un fuerte impacto entre los trabajadores según su nivel de cualificación, que fue mucho más pronunciado en los salarios mejor remunerados del mercado laboral. La caída del salario real de los trabajadores cualificados entre 1968 y 1980 fue de un 4,4\% acumulativo anual, en tanto que la de los trabajadores semicualificados fue del $5,1 \%$ anual.

Durante la década perdida de 1980 en materia económica, pero especialmente con la profundización del proceso de reprimarización y liberalización de la economía uruguaya ocurrido a partir de los años noventa, se revirtió la anterior caída de la brecha salarial por cualificación. Este cambio se debió, por un lado, a la reorientación de la producción manufacturera hacia los bienes agrarios con escaso valor agregado y con una demanda de trabajo poco cualificado y de baja remuneración (Amarante, 2005). Por otro lado, a un incremento de la demanda de trabajadores dentro del sector de los servicios, 
con una gran dispersión en el tipo de mano de obra requerida. Se combina en este sector un fuerte incremento de trabajadores altamente cualificados y remunerados, orientados al sector de los servicios financieros y servicio a las empresas, y un fuerte aumento de los servicios personales y sociales, con menores requerimientos de cualificación y una baja remuneración salarial.

La desregulación y los cambios estructurales a favor de una mayor eficiencia implicaron recortes salariales, reducción de la participación salarial en el PIB y una flexibilización drástica del mercado laboral (Marinakis, 2005). Todo esto condujo a un aumento de la desigualdad global.

Este nuevo patrón de desarrollo, que incluye a todos los países de América Latina y también a los países desarrollados, significó para Uruguay una modificación no solo en la demanda de trabajadores más cualificados en determinados sectores productivos antes mencionados, sino que involucra también una segmentación de la oferta de trabajadores, con un incremento de años de estudio significativo para ciertos sectores de la sociedad y un enlentecimiento de los niveles educativos para otros, en especial para los niveles socioeconómicos medios y bajos (Bértola et al., 2010; Cardozo, 2008; Filgueira et al., 2006; Amarante, 2005)

El inicio del nuevo siglo estuvo marcado por el impacto de una profunda crisis en el año 2002 que, si bien tuvo su origen en el sistema financiero, puso en evidencia la fragilidad del patrón productivo vigente hasta el momento. Los resultados en el mercado laboral fueron dramáticos e impactaron por igual a todos los trabajadores (Marinakis, 2005). Si bien la recuperación de la economía se dio antes, ya para el año 2005 el mercado laboral comenzó a recuperarse, lo que se explica en parte por los cambios ocurridos en materia de políticas productivas y sociales que se iniciaron con la instauración de un Gobierno socialdemócrata a partir de ese año. A pesar de que este trabajo abarca escasos años de dicha recuperación y si bien no pueden destacarse cambios significativos en la demanda u oferta de trabajo en la economía, la reactivación de la negociación colectiva, abandonada desde los años ochenta, la recuperación de los niveles de empleo anteriores a la crisis y el aumento del PIB han contribuido a la recuperación de los salarios en su conjunto (Notaro et al., 2011). Sin embargo, esto ocurre en un contexto en el que los sindicatos necesitan ajustar su participación de acuerdo con la transformación del mercado laboral, la reducción del sector industrial y un aumento del sector de servicios y el trabajo independiente.

\section{Observaciones finales}

Este documento ofrece una visión de largo plazo de la evolución salarial uruguaya (1918-2010) basada en cuatro series salariales anuales de categorías ocupacionales de diferentes niveles de cualificación.

Una de las ventajas de este enfoque es la posibilidad de contar con estimaciones primarias sobre la evolución de los salarios relativos, lo que ha permitido analizar las trayectorias salariales en las diferentes etapas de desarrollo económico y aproximar una respuesta a las siguientes preguntas: ¿cuáles son los momentos de cambio y continuidad?, ¿ cómo de fuerte es el vínculo entre el cambio estructural y la respuesta del mercado laboral?

La evidencia respalda nuestro conocimiento previo sobre la disminución de la desigualdad en Uruguay hacia mediados del siglo xx. Al mismo tiempo, destaca nuevas tendencias específicas del mercado de trabajo. En particular, la reducción de la brecha salarial a mediados de siglo se explicó, principalmente, por la disminución relativa de los salarios de los trabajadores cualificados respecto de los salarios de los trabajadores semicualificados y no cualificados. Los salarios de los trabajadores rurales no cualificados muestran un estancamiento persistente a lo largo de todo el período. Aunque el tipo de mano de obra rural en el sector agrícola uruguayo no exigía habilidades especiales, los resultados nos permiten suponer que, entre otros factores, el aislamiento geográfico de los trabajadores y su escaso poder de negociación puede haber contribuido negativamente en sus salarios.

Para leer los diferentes resultados observados en términos de brecha salarial, recurrimos a una combinación de tres dimensiones implícitas que pueden explicar los cambios observados en esta: el aumento de los niveles de educación en la población de trabajadores, los altibajos de la demanda de mano de obra calificada en diferentes modelos económicos y factores institucionales.

A partir de los niveles salariales alcanzados, pudimos confirmar que, si bien los niveles de educación de toda la población mejoraron, el proceso de industrialización en Uruguay (1930-1970) no fue intensivo en la demanda de trabajo cualificado. El incremento de años de estudio en el promedio de la población a lo largo del período coincide con un descenso en la brecha salarial entre trabajadores cualificados y semicualificados. Esto se explica tanto por una significativa mejora salarial de los semicualificados como por una disminución relativa del premio a la cualificación. Esta situación se revierte, sin embargo, a finales del siglo xx, con un aumento de la brecha entre todos los trabajadores y, en especial, con una distancia significativa de los más cualificados respecto al resto. Nuestra explicación para ello apunta a un reajuste de la estructura productiva del país en el marco de un nuevo paradigma tecnoeconómico que desarrolla especialmente el sector de los servicios. Este aumento de la demanda de trabajo cualificado en algunas ramas del sector servicios encontraría una insuficiente oferta de trabajo con las habilidades específicas requeridas.

Los arreglos institucionales para la fijación de los salarios en el Uruguay han tenido un papel relevante. Una mirada de largo plazo muestra que los cambios de tendencia en la evolución de los salarios relativos están asociados directamente a los momentos de existencia o ausencia de mecanismos de regulación salarial. Durante el proceso de industrialización, la regulación estatal y el proteccionismo contribuyeron a la mejora de los salarios y a reducir la desigualdad. En el período abierto y liberal, posterior a los años setenta, la represión sindical primero, y la desregulación y la flexibilidad del mercado laboral después, contribuyeron a generar un aumento en la brecha salarial. En este período no solo se asiste a una pérdida de espacios de negociación tripartita de los salarios, sino que, además, el desmantelamiento progresivo del sector secundario y el incremento del sector de los servicios cambiaron las reglas de juego para los trabajadores, provocando una menor articulación sindical y una pérdida de poder negociador de este grupo para la fijación de las remuneraciones.

Un último aspecto destacable de esta mirada comparada de la evolución salarial por cualificación es su capacidad explicativa sobre el comportamiento de la desigualdad global en el país. Si bien nuestros datos no permiten medir cuantitativa- 
mente el impacto de la desigualdad salarial ${ }^{2}$ en los cambios de rumbo en materia de equidad, puede observarse que las diferencias en las retribuciones de los trabajadores van en sintonía con la desigualdad general. Los momentos en los que el Uruguay mostró un descenso en los niveles de desigualdad son aquellos en los que las diferencias salariales entre trabajadores se redujeron y lo hicieron, además, por mejoras relativas en el salario de los semicualificados y no cualificados más que por la caída de los salarios de los más cualificados. El aumento de la desigualdad a partir de los años setenta coincide también con una caída drástica del salario de todos los trabajadores, que muestra una paradójica equidad en la pérdida salarial de toda la mano de obra. Sin embargo, la reversión de esta tendencia en materia salarial irá de la mano de un aumento en la desigualdad entre los trabajadores, la que se ha mantenido hasta entrado el siglo xxI.

Hay muchos aspectos a seguir en esta línea de investigación. Entre ellos sería importante incluir en el análisis no solo la estructura productiva, sino también la evolución de la pirámide de población, la incorporación de las mujeres al mercado de trabajo, la segmentación del mercado de trabajo y, a nivel micro, los cambios en tipo y composición de las empresas.

\section{Fuentes}

Archivo Establecimiento Bodega Santa Cruz, Facultad de Humanidades y Ciencias, UDELAR.

Establecimiento Estancia San Pedro del Timote, Archivo General de la Nación.

Oficina de Estadísticas Agropecuarias (DIEA) del Ministerio de Ganadería, Agricultura y Pesca (MGAP).

Archivo Campomar y Soulas, Facultad de Ciencias Sociales, UDELAR. LAR.

Archivo empresa Swift, Facultad de Ciencias Sociales, UDE-

Ministerio de Obras Públicas. Presupuesto Nacional.

Encuesta Continua de Hogares (INE).

Presupuesto Nacional. Datos bianuales. Registro Nacional

de Leyes y Decretos.

Encuesta de Octubre de la OIT (parte I y II).

\section{Bibliografía}

Acemoglu, D. (2003). «Cross-Country Inequality Trends», The Economic Journal, 113 (485), F121-F149.

Alvarez Scanniello, J. (2015). Instituciones, cambio tecnológico y productividad en los sistemas agrarios de Nueva Zelanda y Uruguay: patrones $y$ trayectorias de largo plazo [tesis]. Montevideo: UDELAR, FCS. Disponible en: https://hdl.handle.net/20.500.12008/8022.

Amarante, V. y Arim, R. (2005). «El mercado laboral: cambios estructurales y el impacto de la crisis, 1986-2002", en Uruguay, empleo y protección social. De la crisis al crecimiento. Capítulo II.

Anderson, E. (2001). «Globalization and wage inequalities, 1870-1970», European Review of Economic History, 5, 91-118.

Antía, F. (2001). La economía uruguaya en 1985-2000: políticas económicas, resultados y desafíos. Instituto de Economía. Documentos de Trabajo, DT 4/01. Montevideo. Disponible en: https://hdl.handle. net/20.500.12008/4268.

\footnotetext{
${ }^{2}$ Hay investigaciones que están haciendo importantes avances en este sentido, pero se encuentran todavía en proceso de elaboración (Sinisclachi, Willebald. IECON)
}

Ardente, A. Diaz, F. y Rossi, T. (2004). Crecimiento económico y distribución del ingreso en Uruguay [grado]. Montevideo: UDELAR, FCS.

Astorga, P. (2017). Real Wages during Economic Development in Latin America. Researchgate. Disponible en: https://www.researchgate. net/publication/312384063.

Azar, P. y Fleitas, S. (2009). El manejo del gasto público y la protección social: el caso uruguayo en el siglo xx. DT, 9. Montevideo: UDELAR, Instituto de Economía, Facultad de Ciencias Económicas y Administración.

Bertino, M., Bertoni, R., Tajam, H. y Yaffé, J. (2001). «La larga marcha hacia un frágil resultado. 1900-1955», en Nahum, B. (ed.). El Uruguay del siglo xx. La economía. Montevideo: Banda Oriental, vol. 1, pp. 9-55.

Bértola, L. (1991). La industria manufacturera uruguaya, 1913-1961: un enfoque sectorial de su crecimiento, fluctuaciones y crisis. No. 338.4/ B54i. Montevideo: FCS.

Bértola, L. (2005). «A 50 años de la curva de Kuznets: crecimiento económico y distribución del ingreso en Uruguay y otras economías de nuevo asentamiento desde 1870", Investigaciones de Historia Económica, 1 (3), 135-176.

Bértola, L.2016). El PIB per cápita de Uruguay 1870-2015: una reconstrucción. DT, 48. Montevideo: Programa de Historia Económica, FCS. Disponible en: https://hdl.handle.net/20.500.12008/27146.

Bértola, L. y Bertoni, R. (2000). Educación y aprendizaje: su contribución a la definición de escenarios de convergencia y divergencia. DT, 46. Montevideo: UDELAR, Unidad Multidisciplinaria. Disponible en: https://hdl.handle.net/20.500.12008/4665.

Bértola, L., Calicchio, L., Camou, M. M. y Porcile, G. 1999. Southern Cone Real Wages Compared: A Purchasing Power Parity Approach to Convergence and Divergence Trends, 1870-1996. DT, 19. Montevideo: UDELAR, Unidad Multidisciplinaria. Facultad de Ciencias Sociales. Disponible en: https://hdl.handle.net/20.500.12008/4664.

Bértola, L., Camou, M., Maubrigades, S. y Melgar, N. (2010). «Human Development and Inequality in the 20th Century the Mercosur Countries in a Comparative Perspective.», en Coastworth, J., Salvatore, R. y Challu, A.(eds.). Living Standards in Latin American History. Height, Welfare, and Development, 1750-2000. Harvard: Harvard University Press, pp. 197-233.

Betrán, C. y Pons, M. (2003). "Skilled and Unskilled Labour Wage Differentials and Economic Integration, 1870-1930", in Papers, W. (ed.). 5th European Historical Economics Society Conference. Madrid.

Boyer, R. (1994). «Do Labour Institutions matter for Economic Development? A "Regulation" Approach for the OECD, Latin America and an Extension for Asian", in Rodgers, G. (ed.). Workers, institutions and economic growth in Asia. Ginebra: International Institut for Labour Studies, pp. 25-112.

Bucheli, M. (1992). Los logros educativos y los niveles de ingreso. DT, 3/92. Montevideo: UR. FCS-DE. Disponible en: https://hdl.handle. net/20.500.12008/1966.

Bucheli, M. y Furtado, M. (1998). Flexibilidad del mercado de trabajo en Uruguay. Montevideo: Oficina de la CEPAL.

Camou, M. (2010). Las Instituciones del mercado de trabajo en dos ramas de la industria uruguaya, 1900-1960 [tesis]. Montevideo: UDELAR, FCS. Disponible en: https://hdl.handle.net/20.500.12008/8027.

Camou, M. (2020). "Mercado de trabajo e inmigración en la I Globalización: Uruguay y comparaciones regionales», Revista Uruguaya de Historia Económica, 17 (17), 10-24.

Camou, M. y Maubrigades, S. (2005). «La calidad de vida bajo la lupa: 100 años de evolución de los principales indicadores», Boletín de Historia Económica, 4, 51-63.

Card, D., Lemieux, T. y Riddell, W. C. (2003). Unionization and Wage Inequality: A Comparative Study of the U.S, the U.K., and Canada. Working Paper Series, NBER, w 9473.

Cardozo, S. (2008). Políticas educativas, logros y desafíos del sector en Uruguay 1990-2008. Cuadernos de la ENIA, Políticas Educativas. Montevideo: Ministerio de Desarrollo Social.

Davrieux, H. (1970). Salarios y acción sindical en el Uruguay, industria manufacturera, construcción y gobierno central. No. M-931. Montevideo. 
Diario Oficial (1939). Informe de la comisión investigadora sobre condiciones de vida, salario y trabajo de la clase obrera. Cámara de Representantes. Núm. 98011939. Montevideo.

Espino, A. (2011). «Evaluación de los desajustes entre oferta y demanda laboral por calificaciones en el mercado laboral de Uruguay», Revista de Economía del Rosario, 14 (2), 99-133.

Espino, A. (2004). El desempleo en el Uruguay 1991-2002. Documentos de Trabajo FCEA-IE, DT 01/04. Montevideo. Disponible en: https://hdl.handle.net/20.500.12008/4292.

Federico, G., Nuvolari, A., Ridolfi, L. y Vasta, M. (2021). «The race between the snail and the tortoise: skill premium and early industrialization in Italy (1861-1913)», Cliometrica. 15 (1), 1-42.

Filgueira, F. y Alegre, P. (2008). El sistema de protección social y de relaciones laborales en Uruguay: balance y perspectivas (1985-2009). Documento de Trabajo del IPES, 13. Montevideo.

Filgueira, C., Fuentes, A. y Rodríguez, F. (2006). «Viejos instrumentos de la inequidad educativa: repetición en primaria y su impacto sobre la equidad en Uruguay» en PREAL. Educación y brechas de equidad en América Latina. Santiago, tomo II.

Galor, O. y Tsiddon, D. (1997). «Technological progress, mobility, and economic growth", The American Economic Review, 87 (3), 363-382.

Goldin, C. y Katz. L. (1999). «Human Capital and Social Capital: The Rise of Secondary Schooling in America, 1910-1940», The Journal of Interdisciplinary History, 29 (4), 683-723.

Grantham, G. (1994). «Economic History and the Evolution of Labour Markets», en Grantham, G. y MacKinnon, M. (eds). Labour Market Evolution. London: Routledge, pp. 1-26.

Jacob, R. (1984). Crisis y mercado de trabajo: una aproximación a la problemática de los años veinte y treinta. No. 331.12/J15c. Montevideo: UDELAR, FCS.

Jacob, R. (1981). Breve historia de la industria en Uruguay. Montevideo: Fundación de Cultura Universitaria.

Katz, L. y Margo, R. (2013). «Technical Change and the Relative Demand for Skilled Labor: The United States in Historical Perspective», $\mathrm{Na}$ tional Bureau of Economic Research, (January), 1-71. Disponible en: $10.3386 / \mathrm{w} 18752$.

Lara, C. (2012). Desempeño de la productividad sectorial de la industria manufacturera uruguaya en una perspectiva comparada (1978-1997) [máster]. Montevideo: UDELAR, FCS. Disponible en: https://hdl. handle.net/20.500.12008/7720.

Lewis, W. (1954). «Economic Development with Unlimited Supply of Labor», Manchester School of Economics and Social Studies 28 (2), 139-191.

Macadar, L. (1992). Restauración democrática y política económica. Uruguay 1985-1989. Montevideo: Ediciones de la Banda Oriental.

Marinakis, A., Reinecke, G. y Velasco, J. (2005). «Desafíos para las instituciones y políticas en un mercado laboral cambiante». En Uruguay, empleo y protección social. De la crisis al crecimiento. Santiago de Chile: OIT, capítulo I.

Melgar, A. y Cancela, W. (1985). El desarrollo frustrado: 30 años de economía uruguaya 1955-1985, No. 330 MELd. Montevideo.
Millot, J., Silva, C. y Silva, L. (1973). El desarrollo industrial del Uruguay; de la crisis de 1929 a la posguerra. Montevideo: UDELAR, FCE.

Mincer, J. (1996). Changes in wage inequality, 1970-1990. NBER Working Papers, 5823. National Bureau of Economic Research.

Moraes, M. I. (2003). «Crecimiento, tecnología y competitividad en la ganadería uruguaya, 1870-1930», en Histórias Regionais do Cone Sul, Santa Cruz do Sul. Brasil: EDUNISC.

Mordecki, G. (2017). Uruguay en democracia: treinta años de evolución económica (1985-2015). DT, 8/17. Montevideo: UDELAR, FCEE. Disponible en: https://hdl.handle.net/20.500.12008/18994.

Mordecki, G. (coord.). (2015). Crisis, recuperación y auge: 15 años de política económica en Uruguay (2000-2014). Montevideo: Instituto de Economía, Facultad de Ciencias Económicas y de Administración, Universidad de la República Montevideo.

Mourat, O. (1969). «La inmigración y el crecimiento de la población del Uruguay. 1830-1930», en Cinco perspectivas históricas del Uruguay moderno. Montevideo: Fundación de Cultura Universitaria.

Notaro, J. (1984). La política económica en el Uruguay, 1968-1984. Montevideo: EBO.

Notaro, J., Quiñones, M., Senatore, L. y Supervielle, M. (2011). Las políticas públicas en la reestructura del mundo del trabajo: Uruguay 2005-2009. Montevideo: Ediciones Universitarias.

Notaro, J., Fernández, C. y Sörensen. M. (2012). «Los salarios en la industria manufacturera. Uruguay, 1946-1955», Investigaciones de Historia Económica, 10 (1), 57.67.

Notaro J. y Fernández, C. (2012). «Los impactos de los Consejos de Salarios en el Uruguay», Revista Uruguaya de Historia Económica, 2 (2).

Olesker, D. (2001). Crecimiento y exclusión: nacimiento, consolidación y crisis del modelo de acumulación capitalista en Uruguay (1968-2000). Montevideo: Ediciones Trilce.

Piñeiro, D. y Moraes, M. I. (2008). «Los cambios en la sociedad rural durante el siglo xx», El Uruguay del Siglo xx, 3, 105-136.

Rodgers, G. (1994). «Institutional Economics, Development Economics and Labor Economics», en Rodgers, G. (ed.). Workers, institutions and economic growth in Asia. Ginebra: International Institution of Labor Studies, pp. 124.

Siniscalchi, S. y Willebald, H. (2018). Functional distribution in Uruguay by GDP sectors 1908-2014. Seminario de Investigación, Programa de Historia Económica. Disponible en: https://cutt.ly/fR5aOZZ.

Supervielle, M. y Quiñones, M. (2000). «La instalación de la flexibilidad en Uruguay", Sociologias, 4, 20-65.

Van Zanden, J. L. (2009). «The Skill Premium and the «Great Divergence». European Review of Economic History, 13 (1), 121-153.

Williamson, J. (1996). «Globalization and Inequality then and now, the late 19th and late 20th Century compared», The Journal of Economic History, 56 (2) , 277-306.

Wood, A. (1998). «Globalization and the Rise of Labor Market Inequalities», The Economic Journal, 108, 1463-1482. 